\title{
Zwischen Krieg und Frieden
}

\section{Eine Auseinandersetzung mit dem Werk von Ekkehart Krippendorff}

\section{Eine große Autobiografie und ein paar Irritationen}

Am 12. Juli 2012 war in der Abteilung Literatur der Süddeutschen Zeitung eine Rezension wie folgt überschrieben: »Autobiographien von Akademikern sind meist von erschütternder Belanglosigkeit. Dem kritischen Politologen und Pazifisten Ekkehart Krippendorff aber gelingt mit seinen >Lebensfäden ২ ein fast klassisch zu nennender Bildungsroman der Nachkriegszeit«. Ja, welch ein schönes Buch über das Leben eines großen Intellektuellen! Ein Buch, dessen Lektüre mich im doppelten Sinne mitgenommen hat als eine Biografie, die weit über die Person ihres Autors hinausreicht (Krippendorff 2012). Mitgenommen einmal zunächst in mein eigenes Nachkriegsleben (Jg. 1945): in die Auseinandersetzung mit der NS-Zeit, die zum Teil mit denselben Filmen begann, dann in die Marxismus-Rezeption und die Studentenbewegung, in meine Jahre in der Aufbauphase der Friedens- und Konfliktforschung und später in die schwierigen internen Diskussionen über humanitäre Interventionen zwischen »radikalen « und »relativen « Pazifisten. ${ }^{1}$

Krippendorff, der 1934 in die Zeit des Nationalsozialismus hineingeboren wurde und sich als »Pimpf« wie fast alle seine Altersgenossen völlig mit dem Regime identifizierte, hat noch die Anfänge der Politikwissenschaft im Nachkriegsdeutschland miterlebt - er war wahrscheinlich der erste Vollpolitologe seiner Generation und wurde später in Berlin zu einem der führenden, mit Rudi Dutschke befreundeten Aktivisten der Studentenbewegung und zu einem Mitbegründer der Friedensforschung. Für mich zählt er neben Ernst-Otto Czempiel und Dieter Senghaas, bei denen ich friedensforscherisch gleichsam aufgewachsen bin, zu den drei großen Vätern oder inzwischen Großvätern der Disziplin.

Krippendorff hat einige Jahre in den USA studiert und gelehrt und fast ein Jahrzehnt in Italien - und über beide Länder Bücher und Aufsätze geschrieben. Rückblickend (Krippendorff 2012: 313-315) sieht er in den bürgerkriegsähnlichen Auseinandersetzungen in Bologna, deren Zeitzeuge er im März 1977 wurde, den Keim

1 Für Anregungen und Kritik danke ich der Herausgeberin und den Herausgebern, außerdem Martin Altmeyer, Dieter und Eva Senghaas, Heinz Kluss und meiner Frau Irene Krell. 
für seinen späteren unbedingten Pazifismus, die Ablehnung jeder Art von Gewalt, obwohl er als marxistisch orientierter Linker revolutionären Perspektiven in der Dritten Welt bis in die 80er Jahre noch einiges abgewinnen konnte. ${ }^{2} \mathrm{Zu}$ den USA hat er seit dem Vietnam-Krieg - seine Habilitationsschrift über Die amerikanische Strategie (Krippendorff 1970) wurde in einem politischen Intrigenspiel von der Freien Universität in Berlin abgelehnt, später in Tübingen angenommen - ein äußerst kritisches, aber zu keiner Zeit anti-amerikanisches Verhältnis gewonnen. Heute sieht er die amerikanische Gesellschaft als »politisch krank ohne Anzeichen einer Genesung«, aber er spricht auch von der Hoffnung auf die Rückkehr jenes »historischen Amerika[s] « zu sich selbst, wie es nicht nur er sondern mehrere deutsche und europäische Generationen nach dem Zweiten Weltkrieg kritisch aber inspiriert erlebt hätten (Krippendorff 2012: 258).

$\mathrm{Zu}$ Krippendorffs faszinierenden Seiten gehören seine bildungsbürgerlichen Kompetenzen. In der politischen Theorie, angefangen bei der antiken Klassik, ist er ebenso zu Hause wie in der Philosophie und in der Literatur, und zwar in verschiedenen Kulturen. Außerdem ist er ein hervorragender Kenner des Theaters (und ein bekannter Theaterkritiker) und der Musik. Die Intensität, mit der Krippendorff die Verbindungen zwischen Politik und Kunst beobachtet, erforscht und kommentiert, erscheint mir einmalig in der Politikwissenschaft. Als besonders imponierend und berührend habe ich seine selbstkritischen Ausführungen empfunden. Zur Abkehr von einer möglichen Befürwortung revolutionärer Gewalt kommt die Kritik an der chronischen Verharmlosung der Verhältnisse in der DDR. Die politisch-intellektuelle Linke, er selbst eingeschlossen, aber auch die aus der Emigration in die DDR zurückgekehrten Intellektuellen, SchriftstellerInnen, MusikerInnen, Theaterleute und WissenschaftlerInnen hätten die DDR zu ihren Lebzeiten viel zu schonend behandelt und »die Missachtung der Rechtsstaatlichkeit, die Verhinderung einer diskursiven Öffentlichkeit, die Zensur, die politisch gesteuerte Zulassung zur höheren Bildung, aber auch die Umweltzerstörung « viel zu sehr entschuldigt (Krippendorff 2012: 356-361, das Zitat 359).

Sehr offen sind auch die Passagen über die Prägungen durch die NS-Zeit. Obwohl Krippendorff das allzu Persönliche bewusst aus seiner Autobiografie heraushält, geht er ehrlich mit den Nachwirkungen auch im eigenen seelischen Untergrund um.

2 Wenn Krippendorff im Zusammenhang mit dem Pazifismus-Streit in der Friedensforschung 1993 in einem Interview beklagte, dass sich nun auch Befürworter »militärischer Lösungen « in ihren Reihen befänden, was früher »undenkbar « gewesen sei, so war das nicht ganz korrekt; es sei denn, man begreift antiimperialistische Gewalt oder Befreiungskriege als unmilitärisch (siehe z. B. das letzte Kapitel »Imperialismus, Unterentwicklung und revolutionäres Potential« in Krippendorff 1987: 260-275). 
Das betrifft vor allem sein Verhältnis zu Krieg und Zerstörung, in dem Spuren der bellizistischen Perversionen des Nationalsozialismus noch greifbar sind:

»Aber spannend waren diese Wochenschauen in jedem Fall - auch kamen vor allem in den letzten Kriegsjahren immer wieder Bilder mit Hitlerjungen vor, die sich an der Front bewährt hatten: das sollten und wollten wir natürlich auch bald dürfen. [...] Ich empfinde noch immer eine mir sonst schwer erklärliche dunkle Faszination von Kriegs- und Dokumentarfilmen aus dem Zweiten Weltkrieg, die zwar objektiv den Schrecken und das Grauen des Zerstörens und des Tötens zeigen, die bei mir aber subjektiv keine Schockwirkungen auslösen, sondern eine Art Bestätigung oder Wiederholung von Kindheitswahrnehmungen darstellen, zu denen die Psyche sozusagen masochistisch zurückkehrt« (Krippendorff 2012: 34).

Krippendorffs Autobiografie hat freilich bei mir auch die eine oder andere Irritation ausgelöst. $\mathrm{Zu}$ diesen Irritationen gehört seine Diskussion über einen möglichen jüdischen Widerstand gegen die NS-Herrschaft in Deutschland, in die auch Hannah Arendts Fehldeutungen über die Mitverantwortung der »Judenräte« und (indirekt) über Adolf Eichmann als bloß Anordnungen ausführenden Schreibtischtäter (»Die Banalität des Bösen«) eingegangen sind (Krippendorff 2012: 272-283). Sehr enttäuscht haben mich seine Ausführungen über den Pazifismus-Streit in der Arbeitsgemeinschaft für Friedens- und Konfliktforschung (AFK) in den 90er Jahren. Ich war davon ausgegangen, dass die Verständigungsversuche, an denen er sich beteiligt hatte, zu einer Annäherung oder wenigstens zu einer Art »Waffenstillstand « geführt hätten. ${ }^{3}$ Davon ist in den Lebensfäden leider nichts geblieben; hier erhebt er erneut die alten Korruptionsvorwürfe gegen die »relativen « Pazifisten, also diejenigen, die nicht so radikal staats- und militärkritisch eingestellt sind wie er (Krippendorff 2012: 63-65).

Mitgenommen haben mich die Lebensfäden also auch in einem stärker inhaltlichen Sinne: Denn Krippendorff ist nicht nur ein radikaler Kritiker vergangener und gegenwärtiger politischer Zustände, sondern zugleich ein entschiedener Kritiker seiner beiden Hauptfächer, der Friedens- und Konfliktforschung und der Internationalen Beziehungen, und damit der großen Mehrheit seiner Kolleginnen und Kollegen, die diese Fächer repräsentieren. So habe ich seinen Lebensbericht und seine erneuerte Kritik zum Anlass genommen, mir einen breiteren Überblick über sein

3 Vgl. dazu Grundmann/Hummel (1998), dort vor allem den Beitrag von Ulrike Wasmuht (1998) über die unterschiedlichen Positionen und meinen eigenen Kommentar zu den internen Auseinandersetzungen (Krell 1998). 
Werk zu verschaffen und mich systematischer damit auseinanderzusetzen als ich das in einem kleinen Beitrag schon einmal getan habe (Krell 1999). Ich konzentriere mich hier zunächst auf den Kern seiner Arbeiten zur Friedensforschung, insbesondere auf das Verhältnis zwischen Staat, Krieg und Militär (Krippendorff 1985; 1993; 2000). Krippendorffs Positionierungen spiegeln sich in seiner Auseinandersetzung mit den von ihm so genannten »Bellizisten«, was ich in diesem Zusammenhang kurz rekapituliere. Im Anschluss daran gehe ich auf seine Rezeption des Feminismus ein, die in engem Bezug zum Kernthema steht. Zum Schluss zeige ich an seiner Thukydides-Rezeption, dass Krippendorffs Hyperkritik an den Internationalen Beziehungen mit einer charakteristischen Fehldeutung der Geschichte des Peloponnesischen Krieges beginnt. ${ }^{4}$ Bevor ich zu den kontroversen Themen komme, gebe ich jedoch zunächst einen Überblick über Krippendorffs Oeuvre.

\section{Ein faszinierendes Lebenswerk}

Krippendorff ist ein außergewöhnlich produktiver Mensch. Die in einer Art Festschrift zu seiner Emeritierung angehängte Bibliografie von 1999 umfasste damals schon 18 Monografien, 15 Editionen und jeweils um die 80 Beiträge zu Sammelbänden bzw. in Zeitschriften (Greven/Jarasch 1999: 427-441). Da sind die unzähligen politischen Zeitungsartikel oder seine Theaterkritiken noch gar nicht inbegriffen. Ebenso beeindruckend ist die schon erwähnte Breite dieser Publikationen. Da wäre als erste Kategorie »Grundlagen der Politikwissenschaft und der Friedensund Konfliktforschung « zu nennen. Dazu zählt der Sammelband Friedensforschung (Krippendorff 1968), dessen Texte für meine Generation Pflichtlektüre waren. Der zuerst 1972 ebenfalls in der berühmten »Gelben Reihe« erschienene Sammelband Internationale Beziehungen enthält einen modernen Klassiker von Krippendorff selbst, nämlich seinen Beitrag Ist Außenpolitik Außenpolitik? (zuerst: Krippendorff 1963), der zu den älteren Standard-Aufsätzen der Internationalen Beziehungen in Deutschland gehört.

$\mathrm{Zu}$ den großen in einem anderen Sinne grundlegenden Beiträgen zählt Krippendorffs Internationale Politik. Geschichte und Theorie (Krippendorf 1987), eine umfangreiche Monografie von 1987, die in der Tradition einer empirisch reichhaltigen, theoretisch reflektierten und vom Marxismus inspirierten Weltsystemtheorie steht. In der schon erwähnten Habilitationsschrift Die amerikanische Strategie greift er die Tradition der revisionistischen Geschichtsschreibung in den USA auf, aktualisiert sie und spitzt sie zu. Krippendorffs Texte zu William Shakespeare und Johann

4 Eine wesentlich ausführlichere Fassung stelle ich parallel zur Veröffentlichung dieses Beitrags ins Netz: www.gert-krell.de, dort auf der Unterseite »Papiere«. 
Wolfgang Goethe (Krippendorff 1988; $1992 \mathrm{a} ; 1999 \mathrm{a} ; 2008)$ sind den von ihm verehrten und als politisch äußerst relevant analysierten Dichtern gewidmet (wobei Goethe auch als aktiv tätiger Politiker sein großes Vorbild ist). Sie werden von Literaturwissenschaftlern sehr gelobt; in der Politikwissenschaft haben sie, das beklagt der Autor immer wieder, allerdings bislang kaum eine Rolle gespielt. Dafür mag es Gründe geben, aber seine Duografie über Goethe und Jefferson z. B. sollte zur selbstverständlichen Lektüre einer/eines jeden Politikstudierenden gehören (Krippendorff 2001).

$\mathrm{Zu}$ einer Klasse für sich im doppelten Sinne gehören Die Kunst, nicht regiert zu werden. Ethische Politik von Sokrates bis Mozart (Krippendorff 1999) und Die Kultur des Politischen. Wege aus den Diskursen der Macht (Krippendorff 2009). In beiden Bänden mischt Krippendorff die verschiedensten Themen: von der Entstehung der Politik durch das Theater, dem Peloponnesischen Krieg, Small Is Beautiful und Gandhi über Ästhetische Bildung, den feministischen Blick, die Geburt Europas aus dem Geiste der Mythologie und Freundschaft als politische Kategorie bis zur Idee der Universität, der unerschöpflichen Faust-Parabel und Mozarts Frieden. Das ist nur eine Auswahl aus seinen insgesamt fast 40 hier versammelten Beiträgen. ${ }^{5}$

Besonders herausheben möchte ich seinen Aufsatz USA und Israel. Zwei Projekte der Aufklärung (Krippendorff 2009: 89-100), der zuerst 2002 unter dem Titel Die Vereinigten Staaten und Israel. Projektionsflächen für Hoffnung und Hass erschienen war. Es handelt sich dabei um eine knappe, aber vorzügliche vergleichende kritische Würdigung dieser beiden so unterschiedlichen und doch in vielem ähnlichen Länder. Mit diesen zehn Seiten versteht man die starke Bindung der Vereinigten Staaten an Israel, die man mit der »Israel-Lobby« in den USA nur hoffnungslos unzulänglich erklären kann. Krippendorff selbst hat den Beitrag einmal als einen seiner besten politischen Essays bezeichnet. Ihn als antisemitisch zu lesen, wie das Henryk Broder getan hat, halte ich für abwegig.

5 Zu dieser Gruppe kann man auch noch Krippendorff (1990) rechnen. Seine sehr positive Einschätzung von Wagners politischem Denken, vor allem seiner Geld- und Staatsmachtkritik, die er in der Ring-Parabel verkörpert sieht, teile ich nicht. Ich kann dieses Denken nicht von Wagners Antisemitismus trennen (auch Wagners Kapitalismuskritik war immer antisemitisch konnotiert), nicht von seiner Grandiosität, seinen Gewalt- und Untergangsfantasien, seiner hochgradig affektierten Opernsprache und Theatralik. Köhler (1999) beschreibt in einer materialreichen Monografie Hitler als Wagners getreuen politischen und ideologischen Nachfolger. Auch Thomas Mann, der ein begeisterter Anhänger der wagnerschen Opern war, hatte begriffen, dass in Wagner viel Hitler steckte (siehe Vaget 2010). 


\section{Im Zentrum: Radikalkritik an Staat, Militär und Krieg}

Krippendorff gehört zu den Autoren, die sich und anderen immer wieder Rechenschaft über ihre Grundhaltung geben. Das folgende Zitat ermöglicht einen Einblick, wie er sich politisch und wissenschaftlich einordnet:

»Links sein bedeutet das intellektuelle Vergnügen, immer recht zu haben. [...] Prinzipielle Unzufriedenheit [...] kann sich im analytischen Urteil nicht irren, weil sie einen intellektuellen und psychischen Aggregatzustand kritischer Distanz reflektiert, vor dem keine Wirklichkeit bestehen kann und Anerkennung findet« (Krippendorff 1999: 299).

Nur wenige Politiker wie z. B. Goethe, der zehn Jahre lang eine Art Ministerpräsident im Herzogtum Weimar war, oder US-Präsident Thomas Jefferson finden vor Krippendorffs Urteil Gnade, viele andere werden als entfremdete oder verblendete Machtpolitiker »demaskiert«; nicht nur Shakespeares Mörderkönige, sondern auch Friedrich der Große oder John F. Kennedy. Krippendorffs Texte sind immer für eine Überraschung gut, auch für diejenigen, die sich schon lange mit den Untiefen der Weltpolitik beschäftigt haben. So entlarvt er Che Guevara, diesen »sonst so ungewöhnlich eindrucksvollen Mann«, als »unverantwortlichen Weltuntergangsfanatiker«, der bereit gewesen sei, für eine neue Gesellschaft in Kuba auch einen Atomkrieg in Kauf zu nehmen und die Völker Lateinamerikas auf dem »Altar seines utopischen Weltbilds« zu opfern (Krippendorff 1993: 98, Fn. 11).

Krippendorffs kritische Distanz, vor der keine Wirklichkeit bestehen kann, hat freilich auch eine Kehrseite: die Einebnung dieser Wirklichkeit, in der alle Katzen grau werden. Oder wieder in seinen eigenen Worten: »Der linke Blick auf die Geschichte macht keinen Unterschied zwischen großen und kleinen politischen Führern der Vergangenheit, zwischen fortschrittlichen und reaktionären Fürsten, guten und schlechten Herrschern. Von ihm werden sie alle [...] >ins Nichts« verbannt« (Krippendorff 1999: 295).

\subsection{Staat und Krieg}

Staat und Krieg (Krippendorff 1985), nach Krippendorffs eigenen Angaben sein Hauptwerk, ist zugleich seine idée fixe, das ihn beherrschende Thema. Krippendorff geht davon aus, dass der Staat ein Herrschaftsverhältnis von Menschen über Menschen begründet, das militärisch nach innen und außen abgesichert werden muss. Daraus können sich zwei Pfade zum Krieg ergeben. Einmal ein rationalistischer: Die Staatsräson kann keine emanzipierten Bürger gebrauchen, also setzt sie auf Untertanen, deren Unbildung sie gezielt organisiert, damit diese gegebenenfalls tat- 
sächlich die Interessen der Herrschenden nach außen absichern (Krippendorff 1985: 24). Und sie sorgt für ein Militär, das im Ernstfall diese Interessen auch nach innen, also »unten«, verteidigt. Zum zweiten ein psychologisierender: Staatlich organisierte Herrschaft führt zwangsläufig (auch oder sogar in erster Linie) zur Verdummung der Herrschenden selbst, die in abstrakte Kategorien von Machterhaltung bzw. -erweiterung sozialisiert werden und so nicht nur den Kontakt zur Lebenswirklichkeit und den wirklichen Bedürfnissen ihrer Untertanen verlieren, sondern überhaupt blind werden für Tugend und Vernunft und schließlich sogar ihre eigenen Machtinteressen schädigen. Auch wenn Krippendorff den Herrschenden »Momente der politischen Vernunft« zutraut (Krippendorff 1985: 329), bleibt der Zusammenhang von Militär bzw. Gewalt und Staat doch unauflöslich; der Staat als »Terrain der Torheit produziert politische Unvernunft zwangsläufig und systematisch« (Krippendorff 1985: 14).

In seinem drei Jahre später erschienenen Versuch über Goethes Politik (Krippendorff 1988) greift Krippendorff das Grundthema wieder auf. Neu gegenüber Staat und Krieg ist die Perspektive des Gegenentwurfs: das kleine absolutistische Weimar als »historisches Alternativprojekt« (Krippendorff 1988: 35), das Experiment einer vernünftigen, menschendienlichen Sachpolitik als reale politische Alternative zur »prätentiösen und prestigiösen Machtpolitik « (Krippendorff 1988: 58) von Krieg, Eroberung und Expansion. Der Kern der Argumentation aber ist derselbe:

»Dieses 〉höchste Glück ‘ [nämlich wirklich Herren über die Mittel zu unseren Zwecken zu sein; Anm. GK] ist in der Politik [bzw.] innerhalb der Politiksphäre einer verstaatlichten Gesellschaft nicht möglich. Hier heiligt bekanntlich immer schon der Zweck die Mittel, ist Unvernunft eine Tugend, herrscht die Verkehrung der Werte, ist Sachverstand weniger gefragt als Machtinstinkt« (Krippendorff 1988: 90).

Und Weltpolitik bleibt eine »politische Handlungsebene der Unvernunft« (Krippendorff 1988: 89), sie ist »Machtpoker und Risikospiel« (Krippendorff 1988: 73), kurzum: pathologisch.

In der Militärkritik (Krippendorff 1993) schreibt Krippendorff: »Die Wahrheit ist, dass es Kriege geben wird, solange wir in Staaten als bewaffneten Zwangsverbänden eingesperrt sind « (Krippendorff 1993: 119) - oder: »wer die staatliche Organisation der modernen Gesellschaft nicht in Frage zu stellen bereit (oder in der Lage) ist, der hat das Militär- und Kriegsgefahrenproblem nicht wirklich begriffen « (Krippendorff 1993: 178). Ähnlich heißt es weiter vorn: »Die beliebig zitierten Friedrichs [u. a. Friedrich der Große; Anm. GK], Hitlers, Husseins [u. a. Saddam 
Hussein; Anm. GK] regieren uns noch immer (Krippendorff 1993: 35). In Politik in Shakespeares Dramen (Krippendorff 1992 a) lesen wir: »Bis heute werden wir von denen regiert, die in die Fußstapfen der vielen frühen Richards [darunter Richard III., der bekanntlich seine möglichen Konkurrenten schon als Kinder ermorden ließ und dessen Skelett jetzt gefunden wurde; Anm. GK] getreten sind, auch wenn sie das nicht zugeben können und keine Kronen mehr tragen« (Krippendorf $1992 \mathrm{a}$ : 227). Auch Macbeth verkörpert und verwirklicht die dunkle, die »böse« Seite nicht nur des zeitgenössischen, sondern »in letzter Instanz jedes Staates« (Krippendorff 1992 a: 401).

\subsubsection{Logische und empirische Probleme}

Das Problem mit solchen Pauschalaussagen ist die offenkundige und hochgradige Variabilität in der Beziehung zwischen Staat und Krieg und ebenso zwischen nichtstaatlich organisierten Gesellschaften und Krieg. ${ }^{6}$ Die Einwände gegen seine Programmatik lassen sich davon abgesehen auf fünf Fragen konzentrieren: Ist der Staat 1.) wirklich der entscheidende Kriegsgrund, hängt 2.) die Kriegsneigung von Staaten nicht von einer Reihe anderer Faktoren, z. B. der Herrschaftsform ab, und sind 3.) die Mächtigen von heute wirklich noch dieselben wie in Shakespeares Königsdramen oder im Absolutismus? Könnte 4.) sogar ein Mangel an Staatlichkeit der Hauptgrund für kriegerische Auseinandersetzungen sein (z. B. in den aktuellen Bürgerkriegen in der so genannten »Dritten « und »Vierten Welt«)? Und welche Rolle spielen 5.) die »Beherrschten« auf dem Weg vom Frieden zum Krieg; sind sie immer die Getriebenen oder vielleicht auch mal die Treibenden - eine Vorstellung, die Krippendorff völlig fremd zu sein scheint. ${ }^{7}$

\subsubsection{Staat und Krieg: ein ehernes Gesetz?}

Krippendorff unterscheidet selbst z. B. zwischen den Kriegen der römischen Republik und des römischen Imperiums; an anderer Stelle spricht er davon, dass keiner der heutigen Staaten, und »sei seine Außenpolitik auch noch so friedlich « (Krippendorff 1991: 87; meine Hervorh.), auf Militär verzichten wolle. Interessant ist in diesem Zusammenhang auch sein Hinweis auf Indien, wahrlich kein Kleinstaat, das

6 Krippendorff (1985) enthält auch einen Abschnitt über vorstaatliche Kriege, aber die stuft er soweit herab, dass sie zu seiner Hauptaussage passen; die Literaturlage erlaubt jedoch auch andere Einschätzungen.

7 Auf eine sechste Frage, ob Kleinstaaten eine Alternative zum unauflöslichen Zusammenhang von Staat und Krieg wären, kann ich hier nicht eingehen; vgl. dazu aber Senghaas (2010), der auch auf Krippendorff Bezug nimmt, und darauf aufbauend meine Langfassung im Netz (siehe Fn. 4). 
in seinen Anfängen das Projekt einer ent- oder nichtmilitarisierten Außenpolitik verfolgt habe, das erst durch Chinas Eroberung eines »unbewohnten und unwirtlichen Streifens [indischen; Anm. GK] Gebirgslandes « (Krippendorff 2000: 65) zunichte gemacht wurde: »Wenn es nach dem Zweiten Weltkrieg ein hervorragendes außenpolitisches Verbrechen gegeben hat, dann war es diese chinesische Zerstörung der indischen Alternative zur Welt-Machtpolitik (Krippendorff 2000: 62).

Ich will mich hier auf die gravierenden historischen und politischen Unterschiede im Macht- und Gewaltcharakter staatlicher Herrschaft nach innen und nach außen konzentrieren. Sie bilden den Anknüpfungspunkt für die von Krippendorff so häufig kritisierte große Mehrheit in den Internationalen Beziehungen wie in der Friedensund Konfliktforschung, die staatlich organisierte Herrschaft nicht nur als negativ begreifen; von der Frage der Alternative einmal ganz abgesehen. Die zivilisierende Bedeutung eines rechtsstaatlich gezähmten und demokratisch legitimierten und kontrollierten staatlichen Gewaltmonopols kann angesichts von Staatszerfall und ausufernder substaatlicher Gewalt in vielen Bürgerkriegsgesellschaften gar nicht hoch genug veranschlagt werden. Und auch für den zwischenstaatlichen Verkehr gibt es eine Zivilisierungsperspektive, die verregelte und verrechtlichte Beziehungen zum wechselseitigen Nutzen an die Stelle bloßer Macht- und Gewaltpolitik setzt. ${ }^{8}$ Das alles kommt bei Krippendorff kaum vor.

Wie ein kleiner Exkurs in die Kriegsstatistik zeigt, hat sich die Zahl der Kriege zwischen Großmächten seit dem 16. Jahrhundert kontinuierlich verringert, und zwar von 2,6 Kriegen pro Dekade über 1,7 (im 17. Jh.) und 1 (18. Jh.) auf 0,5 (19. Jh.) und 0,2 (20. Jh.). Kriege zwischen Großmächten sind also immer seltener, allerdings auch immer zerstörerischer geworden, was zusammen genommen gewiss nur ein fragwürdiger Trost wäre. Möglicherweise besteht zwischen diesen beiden Tendenzen ein Zusammenhang, sind sie in der nuklearen Abschreckung zwischen Ost und West sogar zusammengefallen. Torbjörn Knutsen, aus dessen Buch The Rise and Fall of World Orders ich diese Überlegungen übernommen habe, diskutiert freilich noch weitere säkulare Trends, die quer zu den großen Hegemonie- und Gewaltzyklen der Neuzeit stehen: die wachsende Interdependenz der Staaten und die Globalisierung der Zivilgesellschaft. Beide könnten ebenfalls für die (partielle) Pazifizierung der Beziehungen zwischen den Großmächten mitverantwortlich sein (Krell 2009: 91-92).

8 Vgl. Krell (2009: 89-92: Exkurs zu Staat und Krieg). Zur Zivilisierung der internationalen Beziehungen hat sich Dieter Senghaas mehrfach maßgeblich geäußert, etwa 2004 oder 2012; vgl. aber auch Müller (2008). 
Eine andere Untersuchung verweist auf die kumulativen Wirkungen des kantianischen Friedens: Demokratien entwickeln intensive Handelsbeziehungen untereinander und beteiligen sich reichlich an internationalen Organisationen (Russett/ Oneal 2001). Die Effekte des republikanischen, des freihändlerischen und des regulatorischen Friedens verstärken sich offenbar wechselseitig. Freilich bleibt das Verhältnis zwischen Demokratien und Autokratien (oder noch nicht voll entwickelten Demokratien in der »Dritten Welt «) brisant, und dazu gibt es eine Menge Kritisches zu sagen. Aber die Wahrscheinlichkeit, dass moderne Demokratien miteinander in militarisierte Auseinandersetzungen geraten, liegt 41\% unter dem Dyaden-Durchschnitt; gemischte Dyaden liegen 73\%, autokratische Dyaden 67\% darüber (Russett/Oneal 2001: 115). Die »kosmopolitische Sphäre« scheint auf die Welt der entwickelten Industriestaaten beschränkt; die meisten Kriege seit 1945 sind Bürgerkriege und finden in der so genannten »Dritten Welt« statt. »Staat und Krieg" könnte also auch zu einem Problem unzureichender Staatlichkeit geworden sein, es sei denn, man wollte alle Kriege in den Entwicklungs- und Schwellenländern der OECD-Welt anlasten. Auf jeden Fall haben wir es mit einem historischen Trend vom great power war zum weak state war zu tun (Krell 2009: 92).

Auch ein grober Blick auf die Kriegsbeteiligungen zeigt erhebliche Differenzen. So fällt z. B. Schweden im 19. und 20. Jahrhundert ganz aus der Kriegsstatistik heraus, und Schweden ist kein Kleinstaat. Darüber hinaus gibt es auffällige Erbfeindschaften, aber auch Erbfreundschaften. Auch das deutet auf komplexere Beziehungsmuster, als die These vom ewig gleichen Zusammenhang zwischen Staat und Krieg suggeriert.

\subsubsection{Der Machtcharakter der Bundesrepublik Deutschland}

Als zwei Extreme auf einem gedachten Kontinuum staatlicher Macht- und Gewaltpolitik nehme ich das nationalsozialistische Regime auf der einen und Krippendorffs Modell Weimar (s. o.) auf der anderen Seite. Wo auf diesem Kontinuum wäre Deutschland heute anzusiedeln? Durchaus seriöse Studien sehen zumindest eine Tendenz weg vom klassischen Machtstaat hin zum Handelsstaat oder sogar zur Zivilmacht. Nun erkennt auch Krippendorff an, dass es in der deutschen Geschichte Veränderungen, ja Brüche gegeben hat. Neu in Deutschland seien anti-autoritäre und pazifistische Tendenzen, neu Herrschafts- und Militärkritik. Aber all das sei weit davon entfernt, die in der »staatlichen Ordnung verdinglichte Gewalt [...] ernsthaft in Frage zu stellen « (Krippendorff 1993: 73).

Wer sind denn heute die politisch Mächtigen in Deutschland? Sind die MdBs Karl Lauterbach, Karl Lamers, Sigmar Gabriel, Petra Pau, Cem Özdemir und Wolfgang 
Bosbach, der Ministerpräsident Winfried Kretschmann, die Ex-Bildungsministerin Annette Schavan, die Justizministerin Sabine Leutheusser-Schnarrenberger oder der Finanzminister Wolfgang Schäuble Menschen, die Politik machen, (nur) um damit Macht über andere Menschen auszuüben? Oder wenigstens die Kanzlerin Angela Merkel? Mit Sicherheit bringt sie ihre KonkurrentInnen nicht um wie Richard III. - aus meiner Sicht eine Differenz ums Ganze, nämlich um Leben oder Tod -, sondern beißt sie allenfalls symbolisch weg, sperrt sie nicht einmal ein.

Nicht zufällig hat der Versuch von Gertrud Höhler, die Kanzlerin als Machtfrau zu enttarnen, so gut wie niemanden überzeugt (Höhler 2012); eine »Herrschende« im Sinne der (meist männlichen) historischen Figuren, die Krippendorff so brillant analysiert, ist Merkel jedenfalls mit Sicherheit nicht. Alle hier genannten VertreterInnen und viele andere aus der heutigen politischen Klasse in Deutschland stehen jener menschendienlichen Sachlichkeit, die Goethe offenbar nicht nur gefordert, sondern auch repräsentiert hat, deutlich näher als den Machtmenschen, in deren Tradition oder Fußstapfen Krippendorff sie immer wieder stellt. Hierzu noch einmal Krippendorff im Wortlaut: »Zu sagen, dass wir eben nicht von Staatsklugen, sondern von Eigenruhm- und Machtsüchtigen, von pathologischen Herren regiert werden, das ist entweder lebensgefährlich oder es wird, im heutigen Normalfall, als schrullige Außenseitermeinung beiseite geschoben« (Krippendorff 1992 a: 218).

\subsubsection{Die Herrschenden und die »Kleinen Leute«}

Wenn es »Herrschende« gibt, gibt es auch »Beherrschte«. In deren Einschätzung schwankt Krippendorff. Vielfach sind sie selbst in die Verblendungen der Eliten eingebunden, wobei nicht immer klar wird, ob diese Einbindung von den Herrschenden gezielt organisiert wird oder Teil eines (unbewussten) gemeinsamen Prozesses ist. Für die erste Variante sprechen etwa Aussagen, denen zufolge die Völker nicht den Krieg wollen, sondern gegeneinander aufgehetzt werden (Krippendorff 1993: 14, 35) - was auch noch offen lässt, ob sie dazu gezwungen oder nur manipuliert werden. In der Tat werden sie von Krippendorff manchmal als das Opfer der Herrschenden beschrieben, denen sie hilflos ausgeliefert seien (z. B. Krippendorff 1992 a: 250), wird die politische Klasse als der eigentliche Motor aller Probleme charakterisiert. So spricht er in einem Artikel über Europa von groß dimensionierten Katastrophen, die von einer leichtfertigen und »ethisch haltlosen Klasse« entweder durch Unterlassung oder durch aktives Tun zu verantworten seien, vom Kampf der Kulturen, den sie gezielt vom Zaun gebrochen hätten, bis zur Klimaveränderung (Krippendorff 2009: 27-28). Ein anderes Mal machen die Beherrschten mit, weil sie von den Herrschenden entpolitisiert oder bestochen wurden oder auch selbst 
»abgedankt « haben (Krippendorff 1992 a: 285, 290, 320). In »Staat und Krieg« überwiegt eine Tendenz, sie als »Komplizen« der Mächtigen zu deuten, die »in pathologisches, verdummtes Denken« eingeübt wurden oder sich haben einüben lassen (Krippendorff 1985: 2). In Shakespeare sieht Krippendorff zwar einen Sympathisanten des Volkes, aber auch einen Warner vor der Anfälligkeit der »Kleinen Leute« für Demagogie, wobei die Demagogen »wohlmeinende Volksvertreter« oder »zynische Techniker der Machtergreifung « sein können (Krippendorff 1992 a: 23).

Manchmal, etwa in den Analysen zu Shakespeares Komödien, erscheinen die »Kleinen Leute« aber auch als Gegenpol zu den Herrschenden, als bloß Leidende, und perspektivisch auch als diejenigen, die alles ganz anders machen würden. So etwa in Measure for Measure, wo »ein Stück Hoffnung « in den unangepassten gesellschaftlichen Randgruppen liegt, die als »kollektive Protagonisten und Gegenspieler der Regierungsmacht « ins Zentrum der Komödie rücken (Krippendorff 2008: 285). Dass nicht nur herrschende Akteure, sondern auch »Normalbürger« autonome Anteile in die Thematik einbringen, und zwar nicht nur explizite Befürwortung von Krieg und Gewalt, sondern sogar, zumindest phasenweise, begeisterte oder aber doch bewusst akzeptierte bzw. schweren Herzens hingenommene Bereitschaft zur (Gegen-)Gewalt, das scheint nur in ganz wenigen Ausnahmefällen in Krippendorffs Texten auf (z. B. Krippendorff 1985: 376).

Das liegt einmal daran, dass es bei ihm so gut wie keine rationale oder legitime Verteidigung mit militärischen Mitteln gibt. Es hat aber auch damit zu tun, dass ihm der Gedanke, die »Herrschenden« könnten eventuell Wünsche oder Bedürfnisse der Beherrschten aufgreifen oder repräsentieren, fern steht; erst recht die Möglichkeit, dass es nicht sie, sondern die Beherrschten sind, die zum Krieg drängen. Belege für die Kriegsbereitschaft der öffentlichen Meinung im Gegensatz zu ihren moderateren politischen Entscheidungsträgern bringt z. B. Walter Russell Mead:

»Administrations were constantly aware that the American people would not permit their government to look weak or to appease foreign governments. Often, indeed usually, the American government was more pacifistic and isolationist than public opinion. At several points in the nineteenth century, the popular pressure for war against Britain and France was almost overwhelming« (Mead 2002: 205).

In diesem Zusammenhang wäre auch auf den Vietnam-Krieg zu verweisen: Hier kann sich die Kritik nicht auf einen Mangel an Öffentlichkeit berufen, auch wenn die Regierungen mit Manipulationen gearbeitet haben. Zunächst skeptisch gegenüber einer erneuten Entsendung amerikanischer Bodentruppen nach Asien, gab sich 
die Öffentlichkeit, nachdem die Entscheidung, den Krieg endgültig zu amerikanisieren, einmal gefallen war, patriotisch und hat ihn dann lange Zeit mehrheitlich unterstützt. Als er sich hinzog und keine schnelle Kriegsentscheidung absehbar wurde, nahm die Opposition gegen die Politik der Regierung zu, wobei jedoch ein signifikanter Teil der öffentlichen Meinung die Kriegführung weiterhin weder für unmoralisch noch für zu massiv, sondern sogar für zu zurückhaltend hielt. Die »schweigende Mehrheit« vollzog keineswegs eine pazifistische Wende, »unannehmbar war allein der entgangene Sieg «; beim so genannten Vietnamsyndrom ging es also nicht um Selbstbeschränkung, sondern um das Gegenteil, um die »Aversion gegen militärische, politische und juristische `Selbstfesselung «" (Greiner 2007: 545).

Es liegt aber auch daran, dass die politische Psychologie über den ständigen pauschalen Pathologie-Verdacht hinaus keinen systematischen Platz in Krippendorffs Analysen findet. In Versuch über Goethes Politik verneint er sogar ausdrücklich, dass ihr ein Stellenwert für die Diskussion über Staat und Krieg zukomme (Krippendorff 1988: 110). Dabei kann man doch z. B. den Nationalsozialismus gar nicht ohne solche Ansätze begreifen. Die kompensatorische Machtsucht destruktiver Führungspersönlichkeiten ist eines von Krippendorffs zentralen Themen, besonders eindrucksvoll in den Analysen der shakespearschen Königs-Dramen. Aber von der Machtsucht kleiner Leute ist bei ihm selten die Rede. Die nationalsozialistische Herrschaft war, psychologisch oder psychoanalytisch gesprochen, ein »psychosoziales Arrangement«, eine Kollusion (ein Zusammenspiel) zwischen den Machtund Größenfantasien einer extrem nationalistischen (und antisemitischen) Aufsteiger-Elite und den entsprechenden Bedürfnissen bei den Beherrschten. Hitler faszinierte, weil er die Massen über die autoritäre Unterwerfung hinwegtäuschte und die Befriedigung narzisstischer Wünsche nach Größe, Macht und Einssein versprach (und teilweise gezielt organisierte). ${ }^{9}$

In den Lebensfäden beschreibt Krippendorff mit eindrucksvollen Belegen die »tägliche Mobilisierung des dumpfen Volksempfindens« in Berlin gegen die Studentenbewegung in der zweiten Hälfte der 60er Jahre (Krippendorff 2012: 159-161, das Zitat 161). Wer da wen mobilisiert hat, diskutiert er nicht im Detail, aber Verweise auf politisch Verantwortliche, die Springer-Presse oder die Polizeiführung wären nicht unplausibel; wobei freilich zu berücksichtigen ist, dass zu einer Mobilisierung auch Menschen gehören, die sich mobilisieren lassen. Was die Neo-Nazis heute angeht, dürfte es sehr schwer werden, irgendeine Art von Mobilisierung sei-

9 Vgl. Krell (2009: 405-406); ich stütze mich dort nicht nur, aber doch vor allem auf das vorzügliche Buch von Mentzos (2002), insbesondere Teil 4: Das psychosoziale Arrangement. 
tens »der Herrschenden « zu konstruieren; und zwar auch dann, wenn man die dubiosen Verwicklungen des Verfassungsschutzes in Rechnung stellt. Von einer »Kollusion« gar ist so gut wie nichts zu sehen.

\subsection{Das Militär}

In den Lebensfäden heißt es: »Mir begann zunehmend klar zu werden: nicht der Krieg, sondern das Militär als Bedingung der Möglichkeit von Kriegen war das Problem« (Krippendorff 2012: 61). Da es für Krippendorff einen sehr engen, ja geradezu unauflösbaren Zusammenhang zwischen Staat, Militär und Krieg gibt, zeigen sich fast unvermeidlich ähnliche »Einebnungen« bei seinen Analysen des Militärs und des Krieges wie beim Staat und bei den Mächtigen.

\subsubsection{Krieg ist Krieg und (alle) Soldaten sind Mörder?}

So formuliert Krippendorff im Anschluss an ein Zitat des großen Dichters, für Goethes Blick sei nicht die unbestreitbare Tatsache entscheidend, dass Soldaten zum Töten ausgebildet würden, sondern dass »in jedem Uniformträger« die Menschheit getötet, weil mit der militärischen Disziplin die »Schönheit der Seele«, die in jedem von uns ruhe, verkrüppelt werde: »Der Soldat kann nur darum zum >Mörder〈 werden, weil der Mord an ihm selbst zuvor schon begangen worden ist « (Krippendorff 1993: 9). Hier liege der Kern seiner eigenen Militärkritik.

Nun ist die Institution des Militärs nicht nur historisch, sondern auch aktuell aus einer menschenrechtlichen oder demokratietheoretischen Perspektive durchaus problematisch. Die Frage ist wieder, gilt Krippendorffs Charakterisierung immer und für alle gleichermaßen? Die russische Armee ist noch heute ein Schrecken für alle Rekruten, intern wohl doch aber nicht vergleichbar mit der preußischen und der wilhelminischen Armee oder gar der Wehrmacht. Nicht einmal die US-Marines, eine Truppe, die für äußerst brutalen Drill bekannt ist, wäre mit dem Goethe-Zitat und seiner Interpretation durch Krippendorff zureichend beschrieben. Eher zufällig habe ich parallel zu meiner intensiven Krippendorff-Lektüre das Buch von Karl Marlantes (2010) über den Vietnam-Krieg gelesen. Das ist kein pazifistischer, aber alles andere als kriegsverherrlichender Text, sehr nahe am (tödlichen) absurden Theater. Viele der Marines in diesem »Roman « sind gewiss körperlich oder seelisch verkrüppelt aus dem Krieg herausgekommen, wenn sie ihn denn überlebt haben; aber per se »verkrüppelt« als Soldaten im oben zitierten Sinne waren sie nicht. Per se »verkrüppelt« waren auch die amerikanischen Soldaten nicht, die Krippendorff als kleiner Junge als sympathische und freundliche Menschen und unbewusst auch 
als »unsere Befreier und keine triumphierenden Sieger« erlebt hat (Krippendorff 2012: 42-43).

Wo - von Ausnahmen abgesehen - gäbe es z. B. heute noch so dumme Kriegsbegeisterung wie in vielen »rechten« militärischen Schriften der Weimarer Republik, von der NS-Zeit ganz zu schweigen? Krippendorff schildert in seiner Autobiografie am Beispiel eines Briefes von Heinrich Böll vom 29. Dezember 1939 die damals keineswegs ungewöhnlichen verhängnisvollen Illusionen junger Männer über das Soldatentum. Böll spricht davon, wie sehr ihn das »Absolutgestelltsein" des Soldaten anziehe, es reize ihn geradezu. Seine soldatische Sehnsucht sei wirklich, immer an der Front zu sein. Mitte 1944 schreibt er noch von »der elementaren Lust des Mannes am Kampf«. Und Krippendorff fügt hinzu, es falle ihm nicht schwer, sich in einer solchen Haltung als »Verlängerung der ihm als Pimpf eingeübten militarisierten Weltsicht « wiederzuerkennen (Zitate nach Krippendorff 2012: 206-207). Aber seitdem hat sich doch Einiges verändert, und zwar nicht nur bei Heinrich Böll und Ekkehart Krippendorff und nicht nur in Deutschland, wie man z. B. in einem Aufsatz von David H. J. Morgan über Krieg, Militär und Männlichkeit nachlesen kann:

»I have argued that the linkages between masculinity, violence, and the military are rarely, if ever, as straightforward as the dominant model suggests. [...] There are also good reasons to suppose that the linkages have become even weaker and more complex in many modern societies. [...] These interacting changes have underlined the need to see the military as a site for the development of a plurality of masculinities rather than a single, dominant, and highly embodied masculinity« (Morgan 1994: 179).

Aus meiner Krippendorff-Lektüre weiß ich, dass kein geringerer als Präsident Jefferson Krieg »als Notmaßnahme« und »unter bestimmten Bedingungen«, also vor allem zur Verteidigung akzeptiert hat; er hat auch Piraten bekämpfen lassen (Krippendorff 2001: 108-109). Was Hitler-Deutschland betrifft, so scheint Krippendorff - ich habe das angedeutet - Alternativen zum gewaltsamen Widerstand gegen die Eroberungszüge der deutschen Wehrmacht im Kopf zu haben, aber immerhin zitiert er den von ihm (auch von mir) hoch geschätzten scharfen Kriegs- und MilitarismusKritiker Karl Kraus mit einem Satz aus dem Jahre 1935: »Gegen Hitler gibt es nur eines: die Waffe« (Krippendorff 1990: 173, Fn. 14).

Wie kann es dann sein, dass derselbe Krippendorff in seiner Militärkritik schreibt, es stelle sich erst lange hinterher heraus, dass alle Verteidigungskriege Angriffskriege und umgekehrt waren, der Etikettenschwindel gehöre schließlich zum Metier (Krippendorff 1993: 74)? Gleichzeitig verwirft er alle Modelle alternativer oder 
defensiver Verteidigung, so wie sie zeitweise auch in der Friedensforschung intensiv diskutiert wurden. Immerhin lässt Johan Galtung in einem Vorwort zur Militärkritik offen, ob die Zeit für die Abschaffung des Militärs schon gekommen sei oder ob es nicht doch um eine »defensive Verteidigung, anstelle der Aggressivität einer offensiven Verteidigung « gehe (Galtung in Krippendorff 1993: 8). In einem anderen Essay erklärt Krippendorff noch einmal die Differenz zwischen Angriff und Verteidigung für »zweitrangig« (Krippendorff 1992 b) und in der Militärkritik betont er außerdem, Blauhelme seien auch nicht besser als andere Helme. Das einzige was für ihn zählt, ist die einseitige und vollständige Abrüstung: Helme absetzen, nicht blau färben ist seine Devise (Krippendorff 1993: 138-144, 159-180).

Nur wenn Angriff und Verteidigung unter keinen Umständen mehr relevante Unterscheidungskriterien sind, kann man auch alle Soldaten als potenzielle Mörder bezeichnen, wie das Krippendorff wiederholt tut: »Das Militär ist eine Organisation zur Legitimierung durch Gewalt und, offen und direkt ausgesprochen, zum Mord « (Krippendorff 1993: 49). Demgegenüber möchte ich darauf insistieren, dass es einen Unterschied ums Ganze ausmacht, und zwar nicht nur völkerrechtlich, sondern auch politisch und moralisch, ob man als SS-Mann (weiß-)russische Dörfer und ihre Einwohner gleich mit verbrannt hat oder als russischer Jude zu den Partisanen in die Wälder gegangen ist. Eine Friedens- und Konfliktforschung, die die legitimen Sicherheitsbedürfnisse von Gruppen, Völkern und ihren politischen Repräsentanten, auch wenn sie ihr nicht immer einleuchten mögen, nicht ernst nimmt und prinzipiell nicht mehr zwischen lebensgefährlicher Aggression und Notwehr unterscheidet, wird mit ihren Abrüstungsanliegen meines Erachtens nicht weit kommen.

\subsubsection{Militär ist immer Herrschaftsinstrument?}

An dieser Stelle bleibt zu prüfen, inwieweit das Militär Herrschaftsinstrument auch nach innen ist. ${ }^{10}$ Es kann nicht bestritten werden, dass dieser Sachverhalt historisch und aktuell in vielen Fällen eindeutig gegeben ist (Evidenz z. B. bei Krippendorff 1985: 337-345). Nur fehlt auch hier wieder die Differenzierung. Krippendorffs These, Militär sichere Herrschaft auch nach innen, da es im Falle von Revolutionen gegen die eigene Bevölkerung eingesetzt werden könne, haben schon Christoph Weller und Michael Zürn Anfang der 90er Jahre wenigstens kursorisch überprüft, und zwar am Beispiel der Volksrepublik China, der DDR und Rumäniens im Epochenjahr 1989 (Weller/Zürn 1991).

10 Krippendorff bezeichnet übrigens auch die Zöllner, die Bahnbeamten und die Feuerwehr als »aufgeblähte Puppen der Macht« - wegen ihrer Uniformen (Krippendorff 1993: 67). 
Danach konnte in China die Herrschaft der dogmatischen Parteikader gewaltsam gesichert werden, aber auch erst nachdem Truppen aus anderen Landesteilen nach Peking verlegt worden waren. Die zunächst für die Repression der großen Demonstration auf dem Platz des Himmlischen Friedens (mit einer Million Teilnehmern) vorgesehene 38. Armee hatte sich geweigert, gegen die Demonstranten vorzugehen (Weller/Zürn 1991: 98-99). In der DDR waren weder Polizei noch Betriebskampfgruppen und auch das Militär nicht zu »chinesischen Aktionen« gegen die friedlichen Demonstranten bereit. Trotz gegenläufiger Anweisungen der Staatssicherheit erschienen die Betriebskampfgruppen bei der Großdemonstration am 9. Oktober, wenn überhaupt, dann ohne Waffen. In Rumänien hat die Securitate das CeausescuRegime verteidigt, die Armee war jedoch nicht bereit, Gewalt gegen die Bevölkerung anzuwenden (Weller/Zürn 1991: 99-100).

Die Autoren der kleinen Recherche, die man heute um eine Untersuchung der Umstürze in den arabischen Ländern (mit divergierenden Befunden) erweitern könnte, stellen zusammenfassend fest, dass »Gewaltapparate, deren Mitglieder am normalen gesellschaftlichen Leben teilnehmen, nicht oder zumindest entscheidend weniger bereit sind, die Herrschaft einer politischen Klasse, die von weiten Teilen der Bevölkerung nicht (mehr) als legitim erachtet wird, mit Gewalt zu verteidigen « (Weller/Zürn 1991: 101). Krippendorff habe also die Enge des Zusammenhangs von Militär und staatlicher Herrschaft deutlich überschätzt.

\subsection{Pazifismus}

Die Auseinandersetzungen und Diskussionen in der deutschen Friedensforschung zwischen »radikalen« und »relativen« oder, wie Albert Einstein sie genannt hätte, »absoluten« und »entschiedenen « Pazifisten sind in dem schon genannten Tagungsband (Grundmann/Hummel 1998), exemplarisch auch in Offenen Briefen dokumentiert. ${ }^{11}$ Krippendorff haben sie offenbar nicht nachhaltig beeindruckt: In seiner Autobiografie reklamiert er wieder den Begriff des Pazifismus allein für diejenigen, die militärische Gewalt unter allen Umständen ablehnen. Die Fachvertreter der Friedensforschung hätten jetzt (in den 90er Jahren und darüber hinaus) der »Remilitarisierung der Politik und den sie flankierenden wissenschaftlichen Diskursen « hilflos gegenüber gestanden, schreibt er. In bitteren Disputen habe sich die Disziplin gespalten, seien Freundschaften irreparabel beschädigt worden. Und weiter ist die

11 Vgl. etwa die Kontroverse zwischen Dieter S. Lutz/Reinhard Mutz und mir über die Intervention der NATO im Kosovo in der Dokumentation der Frankfurter Rundschau vom 24. März bzw. 17. August 2001 (siehe auch meine Langfassung im Netz, Fn. 4); vgl. aber meinen partiellen Versuch einer Integration der kontroversen Positionen (Krell 1994). 
Rede von einer Bruchlinie zwischen Kriegs- und Nachkriegsgeneration, denn es seien mehrheitlich die Jüngeren gewesen, die »bellizistische Positionen einnahmen und eine Nie-wieder-Auschwitz-Position umstandslos [sic!] mit friedensforscherlicher Rechtfertigung militärischer Interventionen vereinbarten« (Krippendorff 2012: 63-64).

Denjenigen unter den jüngeren »Bellizisten«, die ihre wissenschaftliche Zukunft noch vor sich hätten, unterstellt er Opportunismus. Um ihre beruflichen Karrieren nicht zu gefährden, wollten sie sich nicht zu weit vom realpolitischen Konsens entfernen:

»Das neue, wieder vereinigte Deutschland sollte mit entsorgter NS-Vergangenheit endlich wieder ein gleichberechtigtes - darf man sagen: >gleichgeschaltetes ? [sic!] - Mitglied der Staatengesellschaft werden. [...] Als Friedensforscher grundsätzlich gegen militärische Konfliktlösungen und in jedem Falle gegen eine deutsche Beteiligung an Militärinterventionen zu sein, das hieß sich von diesem realpolitischen Konsens zu entfernen und, in letzter Instanz, auch eben die eigene akademische Karriere in Gremien, bei wissenschaftlichen Bewerbungen oder auch nur für Kongresseinladungen zu riskieren« (Krippendorff 2012: 64-65).

Krippendorffs Polemik gegen den »Bellizismus« seiner Kolleginnen und Kollegen verwundert umso mehr, als er an einer Stelle sogar einräumt, die traditionelle Politik habe mit der militärischen Intervention in Bosnien am Ende »einen gewissen Erfolg « verbuchen können (Krippendorff 1999: 168). Goethe lässt er durchgehen, dass er zwar Kriegsgegner und Antimilitarist, aber kein unbedingter Pazifist war; ihm verzeiht er sogar, dass er in Napoleons Eroberungspolitik anfangs die Chance für eine neue europäische Friedensordnung gesehen, dass er ihn als pacificator Europas missverstanden hat (Krippendorff 1999 a: 80-81, 88-89). Zu Albert Einstein, einem der bedeutendsten Pazifisten des 20. Jahrhunderts, schreibt Krippendorff, niemand habe so nachdrücklich und unermüdlich für eine Politik und Wissenschaft gleichermaßen umfassende »ethische Kultur« geworben (Krippendorff 1999: 323-324, Fn. 7). Dass Einstein den Konflikt zwischen radikalem und relativem Pazifismus in seiner eigenen Biografie verkörpert (Einstein gab 1933 seinen absoluten Pazifismus auf und forderte die Westmächte auf, gegen Hitler aufzurüsten), erwähnt er mit keinem Wort.

Das letzte Wort zum Pazifismus-Streit in der Friedensforschung soll hier jene Nachkriegsautorin haben, die ihn nicht ausgelöst, aber offengelegt hat. Krippendorff nennt »Friedenstruppen « einen Widerspruch in sich, lehnt also auch den Versuch der Friedensstabilisierung mithilfe von Soldaten nach einer kriegerischen Ausein- 
andersetzung ab (Krippendorff 2000: 224). Dazu Ulrike Wasmuht aus dem neuen Nachwort zur ihrem ursprünglichen Vortrag:

»Im Februar 1998 war ich im Rahmen eines soziologischen Einsatzbegleitprojektes in Bosnien und hatte die Gelegenheit, all die Spuren des Krieges mit eigenen Augen zu sehen. Es war für mich, die den Krieg bislang nur aus den Erzählungen der Eltern und Großeltern kennt, etwas anderes, dort selbst zu sein, als zerstörte Häuser in den Medien nur mittelbar zu sehen. Für mich war diese Woche eine prägende Zeit. Ich bin überzeugt, dass die SFOR-Truppe in Bosnien gebraucht wird - m.E. würde der dort labile Frieden ohne sie bald wieder zerstört sein« (Wasmuht 1998: 37).

\subsection{Männer und Frauen - Gewalt, Krieg und Frieden}

Krippendorff gehört zu den wenigen Männern in der Politikwissenschaft, die sich ernsthaft auf die feministische Herausforderung eingelassen haben. Der »feministische Blick «, so schreibt er, enthalte den Vorwurf, dass die Hälfte der Menschheit im Patriarchat durch erzwungene und schließlich auch verinnerlichte Abhängigkeit und Unmündigkeit um wesentliche Lebenschancen gebracht worden, dass ihr im Ganzen gesehen ein gewaltiges Unrecht geschehen sei. Also komme es darauf an, in einer großen Umwertungsarbeit den tatsächlichen Anteil der Frauen an der Geschichte der Menschen bewusst zu machen. Zugleich gehe es darum zu erkennen, was die Asymmetrie zwischen den Geschlechtern und die Universalisierung der männlichen Normen an Kosten für die gesellschaftliche Entwicklung insgesamt verursacht habe (Krippendorff 1999: 352).

Eindrucksvoll konkretisiert Krippendorff diese Überlegungen in seinen Analysen von Shakespeares Dramen und Komödien, deren Lektüre sich offenbar besonders gut für einen feministischen Blick eignet. Denn die hier beschriebenen Herrscher sind Prototypen dessen, was Raewyn Connell als »hegemoniale Männlichkeit« definiert hat (Connell 1995). Zu wahrer Liebe unfähig, streben diese Männer nach der Macht über andere, nach einer fragwürdigen Form von Ehre, für die sie sinnlos ihr Leben opfern. In Shakespeares Troilus und Cressida entscheidet sich Hektor am Ende wider besseres Wissen (er weiß, dass der Raub Helenas unrecht war, und er weiß, dass die Griechen stärker sind und Troja den Krieg verlieren kann) für den Kampf, in dem er schließlich wie ein Hund totgeschlagen wird: »HEKTOR: Seid still, sage ich; meine Ehre hat den Vorrang vor meinem Geschick. Sein Leben hält jeder für wertvoll; aber der Mann von Wert hält die Ehre für weit kostbarer (und) wertvoller als das Leben« (zitiert nach Krippendorff 1992 a: 88). 
Bemerkenswert ist auch Krippendorffs Rezeption (1993: 52-56) von Christel Neusüß' »solidarischer Kritik am Marxismus « aus einer feministischen Perspektive (Neusüß 1989), eine Kritik an der männlich geprägten Gewalttradition in der Arbeiterbewegung und ihrer Theoretiker, gerade auch an Marx und Engels, an der Vergötzung der Gewalt als List der Vernunft, als Produktivkraft, als Motor des historischen Fortschritts. Staat, Krieg und Militär sind auch bei Krippendorff immer männlich kodiert, aber er warnt zugleich vor Stereotypisierungen: Ganz so einfach ist es mit der Verteilung von vermeintlich typisch männlichen bzw. typisch weiblichen Eigenschaften auf die Geschlechter nicht. In Shakespeares Much Ado About Nothing, einer Komödie über den Zusammenstoß der beiden Gender-Welten, erkennt die männerkritische Beatrice am Ende, dass eine rein feminine Welt ohne männlichen Widerpart nicht nur eine unvollkommene, sondern auch eine gefährdete Welt wäre und dass es »echte männliche Werte gibt, die sich nicht zuletzt in der Verteidigung bedrohter und verletzter Frauen zu bewähren haben « (Krippendorff 1998: 186, 176-177).

Der feministische Blick kritisiere auch die Frauen, sagt Krippendorff, und zwar für ihre mitverschuldete Akzeptanz und Internalisierung der eigenen Unterwerfung (Krippendorff 1999: 349). Inwieweit sie Opfer einer männlich dominierten Welt oder an der Gewalt dieser Welt aktiv beteiligt sind, das bleibt jedoch offen. In den Shakespeare-Analysen kommen Frauen als tätige Komplizinnen der Macht vor, wie z. B. Volumnia, die militarisierte Heroenmutter des Coriolan, die ihren Sohn unbedingt an der Macht sehen will und weiß, dass Macht nur über die Männerwelt zu erreichen ist. Und doch nimmt Krippendorff sie in Schutz: In ihrer "perversen Selbstverkrüppelung als Frau und Mutter« bleibe sie letztlich ein »tragisches Opfer der Männergesellschaft«, obwohl sie der eigentliche Schurke des Stücks sei, der »tragisch-böse Motor hinter dem ganzen Politik-Drama« (Krippendorff 1992 a: 255-256).

Von Lady Macbeth, die aus Machtlust und Ehrgeiz ihren Mann zum Mord treibt, schreibt er, sie unterdrücke seiner Karriere zuliebe »ihre eigene Menschlichkeit, ihre Weiblichkeit, ihr Geschlecht in sich «; sie wolle selbst zum Mann werden (Krippendorff 1992 a: 419). Auch Helena, die das Spiel der Männer um Ehre, Sex und Kampf mitspielt, oder Cressida, deren »ganze Kunst in der sexuellen Verführung der Männer « besteht, sind letztendlich Opfer, Objekte der Männerwelt (Krippendorff 1992 a: 93-94). Wirkt hier bei Krippendorff nicht doch ein ähnlicher Reflex wie bei seiner Diskussion des Verhältnisses von Herrschenden und Beherrschten? Auch Frauen haben Optionen; sie müssen die Machtspiele der Männer nicht mitmachen, obwohl sie das, wie Antigone zeigt, unter Umständen teuer zu stehen kommt. Millionen von deutschen Frauen, die Hitler gewählt (1932 waren es in vielen 
Großstädten mehr Frauen als Männer) und ihm auch nach der Machtergreifung weiter zugejubelt, ja ihn teilweise gottähnlich verehrt haben, hätten das, genauso wie Millionen Männer, so nicht machen müssen. Sie hätten sich weitgehend risikofrei anders entscheiden können, auch ohne in offenen Widerstand oder nur Unbotmäßigkeit zu gehen. ${ }^{12}$

Manche Feministinnen sehen nicht zuletzt deswegen die Rolle ihrer Geschlechtsgenossinnen etwas kritischer als Krippendorff. Keine Frage: Frauen sind im Patriarchat systematisch benachteiligt, und die Gewalttäter sind überwiegend Männer sei es aufgrund des (sexualisierten) Herrschaftsverhältnisses zwischen den Geschlechtern oder gesellschaftlicher Arrangements, einschließlich auf Konsens beruhender Arbeitsteilung. Aber Frauen sind nicht nur »Gewaltphantasiteurinnen, Gewaltassistentinnen und Gewaltclaqueurinnen«, sondern auch eigenständige Gewaltakteurinnen, wie Astrid Albrecht-Heide argumentiert hat (Albrecht-Heide 1983). Und Ruth Waldeck $(1989 ;$ 1991) hat in ihren literarisch-psychologischen Analysen der Kassandra und der Kindheitserinnerungen von Christa Wolf herausgearbeitet, wie Frauen Gewaltfantasien und Gewaltbereitschaft an Männer delegieren oder ihre Verstrickung in Gewalt und Rassismus verleugnen.

Radikale Feministinnen argumentieren ähnlich wie Krippendorff, dass es nicht darauf ankomme, den Frauen den gleichberechtigten Zugang zur Männerdomäne Militär zu verschaffen, sondern das männerdominierte Militär überhaupt abzuschaffen. Bei anderen Feministinnen freilich ist die Perspektive einer auf die Defensive ausgerichteten Sicherheits- und Militärpolitik durchaus positiv besetzt (Seifert/Eifler 2003). Was die Einstellungen von Männern und Frauen zu Krieg und Frieden angeht, so rechtfertigen die empirischen Daten nicht die auch analytisch verdächtige Dichotomie »kriegerische Männer« vs. »friedliche Frauen«, aber sie zeigen auch keine völlige Indifferenz der Kategorie Geschlecht. Noch stärkere Bedeutung könnte einem anderen Befund zukommen, nämlich Zusammenhängen zwischen Geschlechterdemokratie und Frieden. Ein Abbau der hierarchisierten Lebensund Gefühlswelten könnte beide Geschlechter für eine größere Friedensbereitschaft und -kompetenz qualifizieren. Dafür sprechen nicht nur aktuelle empirische Studien (Bussmann 2012), sondern auch Krippendorffs Analysen der shakespearschen Dramen und Komödien.

12 Den Mythos, die Frauen seien im Nationalsozialismus nur Opfer gewesen, hat die feministische Diskussion schon lange hinter sich gelassen. Eine Übersicht über den Stand der Forschung gibt Koonz (2008). 


\section{Krippendorff und die Politikwissenschaft}

Ein immer wiederkehrender Vorwurf Krippendorffs gegenüber der Politikwissenschaft lautet, sie rede in ihrer großen Mehrheit den Mächtigen nach dem Mund und lehre nur die klassische Logik der Staaten- und Machtpolitik. Ihre Vertreter sind für ihn fast ohne Ausnahme »hörige Kommentatoren der herrschenden Verhältnisse« (Krippendorff 2000: 111), die Internationalen Beziehungen (IB) produzierten (weitgehend) »Herrschaftswissen für Armlehnstrategen« (Krippendorff 2000: 91). Einigen wenigen »randständigen Einzelkritiker[n] «, deren Stimme kaum mehr durchdringe, stellt er die Klasse des »privilegierten Mainstreams mit verinnerlichter Machtnähe« gegenüber (Krippendorff 1992 c: 5). In seiner Autobiografie zitiert er noch einmal ausführlich aus diesem Artikel über »Den Mann im Grauhelm«, in dem er den »Fall« des Kollegen Hanns-Dieter Jacobsen, der wegen Spionagetätigkeit für die DDR verhaftet worden war, als logische Konsequenz einer Disziplin gedeutet hatte, die ihre Standards längst verraten habe (Krippendorff 2012: 176-178).

Krippendorff ist in Kategorien der IB-Theorie gesprochen ein radikaler »Realist «. In seiner Analyse des shakespearschen King Lear, ein Schlüsseldrama für sein politisches Weltbild, wie er selbst sagt, ist das »Naturgesetz der Politik« der »erbarmungslose Kampf um die Macht« (Krippendorff 1992 a: 440). Nicht zufällig ist "pathologisch « Krippendorffs am häufigsten verwendetes Adjektiv für diese brutale Welt, die außerdem noch aller Wahrscheinlichkeit nach auf ihren Untergang zusteuert. Das Gegenbild dazu, und insofern ist Krippendorff ein ebenso radikaler Idealist, bildet das Reich der Liebe, der Freiheit, der solidarischen Netzwerke, der überschaubaren Gemeinschaft, der Musik und der Literatur. Nicht nur Shakespeares Komödien stehen für diese andere Welt, sondern zumindest für einen Moment verkörpert auch Hamlet die Option des Ausstiegs aus der (Macht-)Politik und der Hinwendung zur Reflexion über eine mögliche alternative Gesellschaft des Denkens, der Kunst und der Philosophie (Krippendorff 1992 a: 382). So könnte man Krippendorff also einen Sozialphilosophen nennen, der aus einer radikal-oppositionellen Haltung und bewusst von jeder Macht abgeschnitten von unseligen Zuständen Zeugnis ablegen und zugleich eine ganz andere Welt entwerfen will, ohne sich um Strategien für schrittweise Reformen der bestehenden Verhältnisse zu kümmern. ${ }^{13}$ Für diese Interpretation sprechen auch die beiden folgenden Selbstzeugnisse.

13 Neben den schon in Fn. 8 genannten Titeln von Senghaas und Müller aus meiner Sicht immer noch mustergültig als Beispiele für »reformistische« Friedensstrategien: Czempiel $(1998 ; 1999)$. 
In einem Beitrag zu Karl Kraus, in dessen radikaler Kriegs- und Militärkritik sich Krippendorff gespiegelt findet, hat er Fragen an den Autor gestellt, die auch an ihn selbst gerichtet sein könnten:

»Verfällt mit diesen und anderen Verurteilungen [...] nicht die Politik überhaupt seinem [i.e. Karl Kraus'; Anm. GK] rigorosen Verdikt? Ist dann nicht alles Politische, jede politische Klasse, jede, auch noch die friedvollste politische Führung \des Teufels $₫$ ? Führt eine solche Kritik nicht notwendig in eine sterile Leere und verurteilt sie sich nicht damit selbst zur Wirkungs- und Folgelosigkeit, die nur überlebt dank ihrer sprachlichen Brillanz und als große Literatur?« (Krippendorff 1990: 167).

Und gleichzeitig holt er sich aus dem Leiden an dieser so scharf zu verurteilenden Welt die - wie ich finde hoch problematische - Berechtigung, die Verantwortlichen dafür bzw. diejenigen, die er für die Verantwortlichen hält, zu entlarven, ja zu hassen, um sich so Erleichterung zu verschaffen:

»Wer wird Kraus ob solchen Hasses wirklich tadeln wollen? Nur der, der nicht selber auch die Erfahrung gemacht hat, dass konkreter, gezielter Hass, dass die leidenschaftliche Verachtung der Herrschenden und aller ihrer mittleren und kleineren Trabanten, Mitläufer, Profiteure und Opportunisten - gewissermaßen als >Kompensation $<$ - das physische so gut wie das psychische Leiden der Menschen dieser unserer Zeit, unser Mit-Leiden ihres kleinen und großen Elends $[\ldots]$ gerade noch erträglich, gerade noch ertragbar macht « Krippendorff 1990: 170-171).

Neben der Tendenz zur Fundamentalopposition liegt ein weiterer Grund für Krippendorffs Hyperkritik an den Internationalen Beziehungen in seiner selektiven Wahrnehmung dieser Teildisziplin, denn nicht einmal die Theorietradition des Realismus lässt sich für seine Sicht in Anspruch nehmen, was ich hier wieder nur an einem Beispiel, allerdings einem sehr wichtigen, dokumentieren kann. Denn Krippendorff sieht in Thukydides' Geschichte des Peloponnesischen Krieges die verhängnisvolle Grundlegung für die Machtorientierung der IB; in meinen Augen eine nicht zufällige Fehldeutung. So schreibt er, Thukydides' klassischer Text sei zum Rechtfertigungsbuch für alle Realpolitiker geworden, zum Teil durch deren Deutungen, zum Teil durch einen »radikalen Reduktionismus« in der Schrift selbst. Denn der griechische Historiker habe das Wesen der Politik als Durchsetzung von Macht so kraftvoll bestimmt, dass sie »nicht nur zu einem analytischen, sondern vor allem zum nicht mehr hinterfragten, handlungsleitenden und -rechtfertigenden $\mathrm{Pa}$ radigma« geworden sei (Krippendorff 2009: 29). Die Chance, aus den falschen 
Lehren des Thukydides auszusteigen, die im Gegensatz zu ihm Dichter wie Sophokles oder Euripides mit ihren kriegskritischen Dramen geboten hätten, sei bis heute vertan worden (Krippendorff 1999: 90-92).

Aber schon der Begriff der Macht ist bei Thukydides nicht eindeutig; es ist also keineswegs offensichtlich, welchen Realismus er vertritt. ${ }^{14}$ Die ungezügelte Verfolgung der eigenen Machtinteressen auf Kosten anderer Stadtstaaten bildete die Grundlage der athenischen Politik für eine Generation und länger. Damit war aber zugleich der Grundstein gelegt für den moralischen und schließlich auch den politischen Verfall im Innern. Thukydides schwebt also offenbar eine maßvolle, eine kluge Variante von Machtpolitik vor (wobei »klug« hier nicht im Sinne Machiavellis oder Richelieus zu verstehen ist). Ein kluger Realismus stellt sich darauf ein, dass es in der internationalen Politik um Macht geht, aber er treibt die eigene Machtpolitik nicht auf die Spitze. Dafür sprechen nach Thukydides sowohl realpolitische als auch moralische Gründe. Aber er ist skeptisch, ob die Politik angesichts der menschlichen Schwächen dauerhaft zu dieser Mäßigung in der Lage ist. Damit bekommt sein Weltbild eine tragische Färbung.

Der berühmte Melier-Dialog, in dem Athen dem Inselstaat Melos anbietet, sich ihm freiwillig zu unterwerfen und mit ihm zu verbünden, andernfalls würde es zerstört, enthält eben nicht nur die unaufgeklärt machtpolitische Argumentation Athens mit dem Recht des Stärkeren. Diese Argumentation, da hat Krippendorff zweifellos Recht, findet sich in der Tat in der Geschichte der Machtpolitik immer wieder. Aber wohlgemerkt: Hier spricht Athen, nicht unbedingt Thukydides. In Krippendorffs Analyse fehlt z. B. die Argumentation von Melos, das Athens Machtanspruch eine andere Programmatik entgegensetzt: das Konzept einer Rechtsordnung, die auch die Schwächeren schützt. Melos begründet diese Rechtsordnung übrigens nicht bloß moralisch, sondern mit dem Nutzen für alle Beteiligten, denn auch der Stärkere könne stürzen und was dann?

Nun könnte sich Krippendorff darauf berufen, dass Athen seinen Machtanspruch durchgesetzt hat: Melos wird zerstört, die erwachsenen Männer werden hingerichtet, Frauen und Kinder in die Sklaverei verkauft. Aber langfristig hat Melos Recht behalten. Denn seine Zerstörung bildet den Auftakt zur Sizilianischen Expedition Athens, deren Scheitern dessen Niedergang einleitet. So ist der Melier-Dialog also gerade kein hohes Lied auf unverhüllte machiavellistische Machtpolitik, wie etwa Friedrich Nietzsche noch meinte, sondern er steht - in den Zusammenhang des

14 Vgl. Krell (2009: 132-133). Neben Thukydides' Original in deutscher Übersetzung und Lebow (2003) stütze ich mich auf Johnson Bagby (1994), Forde (1995), Gustafson (2000) und Welch (2003). 
ganzen Krieges gestellt - für das Scheitern von unkluger Machtpolitik oder - ganz in Krippendorffs Sinne - für die »Torheit der Regierenden«.

Richard Ned Lebow geht noch weiter und bezeichnet Thukydides' Text selbst als eine Tragödie wie die der zeitgenössischen Dramatiker (also wieder eigentlich im Sinne Krippendorffs), nur in anderer Form. Im Kern der Geschichte geht es um den tragischen Helden Athen, der in den vertrauten Zyklus von Erfolg, Selbstüberschätzung, Übermut, Fehlkalkulation und Katastrophe gerät. Im Zuge seines Aufstiegs hat der führende griechische Stadtstaat die alten Konventionen verlassen und sich dem schlechten Vorbild der Perser angenähert. Damit verspielt er das Ansehen, das er sich einst durch den Sieg über die Invasoren erworben hatte. Der Melier-Dialog zeigt den Verfall der attischen Hegemonie, die die Grundregeln aufgeklärter Herrschaft (griechisch: hegemonía) wie Selbstbindung des Hegemons, Rücksichtnahme auf die Interessen der schwächeren Verbündeten sowie die Beachtung ethischer Grundregeln der Zeit aufgegeben hat und sich nur noch auf nackte Machtpolitik (griechisch: archè) stützt. Dieser Art von Machtpolitik setzt Thukydides Besonnenheit, Klugheit, Urteilskraft, Selbstkontrolle, Zurückhaltung und die Beachtung normativer Konventionen entgegen. Das ist nicht nur erfolgreicher, sondern bildet auch die Grundlage für gute und stabile Ordnung, ja für Zivilisation überhaupt (Lebow 2003: Kap. 4).

\section{Schluss}

Ich kenne keinen anderen Lehrer oder Kollegen in der Politikwissenschaft bzw. in der Friedens- und Konfliktforschung, dessen Werk derart widersprüchliche Reaktionen bei mir auslöst wie Ekkehart Krippendorff. Einmal Bewunderung für ein von Umfang und Themenbreite faszinierendes Oeuvre; Bewunderung seiner Weltläufigkeit, seiner Belesenheit, seiner Kunst-, Literatur-, Geschichts-, Politik- und Philosophiekenntnisse, seiner großartigen literarisch-politischen Analysen. Daneben steht bei mir freilich viel Ratlosigkeit über seinen fundamentalistischen Reduktionismus. Staat ist Staat und damit die auf ewig gleiche Zwangsjacke, das auf ewig gleiche Herrschafts- und Kriegsinstrument. Das Militär schlechthin, und zwar bis $\mathrm{zu}$ den Blauhelmen, die Uniform, ist der Feind, nicht »das Böse« (so nennt er selbst einmal den Nationalsozialismus; Krippendorff 2012: 228), das alle anderen, die ja nicht einfach Bellizisten sind, nur weil sie sich schützen wollen, zu verschlingen droht. Der Staat, die Mächtigen von heute sind auch nicht viel anders als die Mordkönige der shakespearschen Dramen; Politik ist und bleibt dumme Machtpolitik und damit letztlich ein schmutziges Geschäft. 
Hinzu kommt Enttäuschung bis Zorn über die Art und Weise, wie Krippendorff mit seinen eigenen Fächern und der Mehrheit seiner Kollegen umgeht, die so gar nicht zu seinen eigenen Anforderungen an demokratischen Diskurs in wechselseitiger Anerkennung passt. ${ }^{15}$ Die Aufteilung der Politikwissenschaft bzw. der Friedens- und Konfliktforschung in ein kleines Häuflein unabhängiger Aufrechter und die Mehrheit derjenigen, die sich opportunistisch oder machtgierig an die Realpolitik verkauft hätten, ist eine selbstgerechte Inszenierung und nicht nur analytisch, sondern auch politisch-praktisch höchst fragwürdig.

Immerhin hat Krippendorff einmal in einem Aufsatz über Abrüstung angedeutet, dass er nicht grundsätzlich gegen Kooperation zwischen »Radikalen« und »Pragmatikern« sei. In einer »Antirüstungsbewegung« dürfe und müsse es Pluralismus geben. Die einen könnten sagen: »ja, Abrüstung ist morgen prinzipiell erreichbar«; die anderen: »)ja gut, aber erst in 100 Jahren «. Jede Position in diesem Spektrum dürfe mit dem legitimen Anspruch auftreten, realistisch zu sein. Alle müssten aber wissen, dass es auf effektive Abrüstung ankomme und nicht darauf, die Rüstung und das Militär lediglich zu kontrollieren und die Gefahren bloß durch technische Lösungen kurzfristig zu entschärfen (Krippendorff 1993: 177-178). So weit geht Krippendorffs Pragmatismus dann doch wieder nicht.

Und ich fürchte, das breite Bündnis zwischen denen, die morgen schon abrüsten wollen, und denjenigen, die mit einem Jahrhundertprojekt rechnen, wird letzten Endes doch sehr schmal bleiben, denn noch einmal: »Wer die staatliche Organisation der modernen Gesellschaft nicht in Frage zu stellen bereit oder in der Lage ist, der hat das Militär- und Kriegsgefahrenproblem nicht wirklich begriffen« (Krippendorff 1993: 178). Die übergroße Mehrheit in der Friedens- und Konfliktforschung, ja selbst in der Friedensbewegung dürfte unter dieses Verdikt fallen. Sie wird mit ihren Forschungen, Projekten und Aktivitäten nicht auf das Absterben des Staates setzen.

15 In der Einleitung zu Shakespeares Komödien schreibt er z. B.: »Die Fähigkeit zum Positionswechsel ist nicht zuletzt eine der Voraussetzungen für das Funktionieren einer demokratischen Gesellschaft [...], denn sie zeigt, dass man bereit und willens ist, Standpunkt, Meinung, Motivation und Urteil eines Anderen - des Mitbürgers auf der Agorà der Entscheidungsfindung - nachzuvollziehen, ernst zu nehmen, zu verstehen, ohne sie deshalb teilen zu müssen « (Krippendorff 2008: 23-24). 


\section{Literatur}

Albrecht-Heide, Astrid 1983: Das Verhältnis von Mädchen und Frauen zur Gewalt, in: Rajewski, Christiane (Hrsg.): Rüstung und Krieg. Zur Vermittlung von Friedensforschung, Frankfurt a. M., 261-273.

Bussmann, Margit 2012: Literaturbericht. Quantitative Studien zu Geschlechtergleichheit und Frieden, in: Zeitschrift für Friedens- und Konfliktforschung 1: 1, 141-154.

Connell, Raewyn 1995: Masculinities, Berkeley, CA.

Czempiel, Ernst-Otto 1998: Friedensstrategien. Eine systematische Darstellung auBenpolitischer Theorien von Machiavelli bis Madariaga, 2. Aufl., Opladen.

Czempiel, Ernst-Otto 1999: Kluge Macht. Außenpolitik für das 21. Jahrhundert, München.

Forde, Laurie Steven 1995: International Realism and the Science of Politics. Thucydides, Machiavelli, and Neorealism, in: International Studies Quarterly 39: 2, 141-160.

Greiner, Bernd 2007: Krieg ohne Fronten. Die USA in Vietnam, Hamburg.

Greven, Thomas/Jarasch, Oliver (Hrsg.) 1999: Für eine lebendige Wissenschaft des Politischen. Umweg als Methode, Frankfurt a. M.

Grundmann, Martin/Hummel, Hartwig (Hrsg.) 1998: Militär und Politik - Ende der Eindeutigkeiten? Zum Wandel institutionalisierter Gewalt, Baden-Baden.

Gustafson, Lowell S. (Hrsg.) 2000: Thucidides' Theory of International Relations.

A Lasting Possession, Baton Rouge, LA.

Höhler, Gertrud 2012: Die Patin. Wie Angela Merkel Deutschland umbaut, Zürich. Johnson Bagby, M. 1994: The Use and Abuse of Thucydides, in: International Organization 48: 1, 131-153.

Köhler, Joachim 1999: Wagners Hitler. Der Prophet und sein Vollstrecker, München.

Koonz, Claudia A. 2008: Geschlecht, Gedächtnis und Geschichtsschreibung. Die

Historiographie zum Dritten Reich und zum Holocaust, in: Hagemann, Karen/

Quataert, Jean H. (Hrsg.): Geschichte und Geschlechter. Revisionen der neueren deutschen Geschichte, Frankfurt a. M., 256-289.

Krell, Gert 1994: Wie der Gewalt widerstehen? Die Frage legitimer Gegengewalt als ethisches und politischen Problem, in: Aus Politik und Zeitgeschichte 44: 2, 29-36.

Krell, Gert 1998: Konfliktfähiger Pazifismus. Ein Kommentar, in: Grundmann/ Hummel 1998, 41-50. 
Krell, Gert 1999: Die Intellektuellen und die Macht - Kritische Anmerkungen zum Korruptionsvorwurf gegenüber der Politikwissenschaft und der Friedensforschung, in: Greven/Jarasch 1999, 107-117.

Krell, Gert 2009: Weltbilder und Weltordnung. Einführung in die Theorie der internationalen Beziehungen, 4. Aufl., Baden-Baden.

Krippendorff, Ekkehart 1963: Ist Außenpolitik Außenpolitik? Ein Beitrag zur Theorie und der Versuch, eine unhaltbare Unterscheidung aufzuheben, in: Politische Vierteljahresschrift 4: 3, 243-266.

Krippendorff, Ekkehart (Hrsg.) 1968: Friedensforschung, Köln.

Krippendorff, Ekkehart 1970: Die amerikanische Strategie. Entscheidungsprozess und Instrumentarium der amerikanischen Außenpolitik, Frankfurt a. M.

Krippendorff, Ekkehart (Hrsg.) 1973: Internationale Beziehungen, Köln.

Krippendorff, Ekkehart 1985: Staat und Krieg. Die historische Logik politischer Unvernunft, Frankfurt a. M.

Krippendorff, Ekkehart 1987: Internationale Politik. Geschichte und Theorie, Frankfurt a. M.

Krippendorff, Ekkehart 1988: »Wie die Großen mit den Menschen spielen«. Versuch über Goethes Politik, Frankfurt a. M.

Krippendorff, Ekkehart 1990: Politische Interpretationen. Shakespeare, Stendhal, Balzac, Wagner, Hasek, Kafka und Kraus, Frankfurt a. M.

Krippendorff, Ekkehart 1992 a: Politik in Shakespeares Dramen, Frankfurt a. M..

Krippendorff, Ekkehart 1992 b: Pazifismus - Bellizismus, in: Jäger, Uli/Birckenbach, Hanne-Margret/Wellmann, Christian (Hrsg.): Jahrbuch Frieden 1993, München, 28-40.

Krippendorff, Ekkehart 1992 c: Der Mann im Grauhelm, in: Die Zeit, 11.12.1992, http://pdf.zeit.de/1992/51/der-mann-im-grauhelm.pdf; 16.3.2013.

Krippendorff, Ekkehart 1993: Militärkritik, Frankfurt a. M.

Krippendorff, Ekkehart 1999: Die Kunst, nicht regiert zu werden. Ethische Politik von Sokrates bis Mozart, Frankfurt a. M.

Krippendorff, Ekkehart 1999 a: Goethe. Politik gegen den Zeitgeist, 2. Aufl., Frankfurt a. M.

Krippendorff, Ekkehart 2000: Kritik der Außenpolitik, Frankfurt a. M.

Krippendorff, Ekkehart 2001: Jefferson und Goethe, Hamburg.

Krippendorff, Ekkehart 2008: Shakespeares Komödien. Spiele aus dem Reich der Freiheit, 2. Aufl., Berlin.

Krippendorff, Ekkehart 2009: Die Kultur des Politischen. Wege aus den Diskursen der Macht, Berlin. 
Krippendorff, Ekkehart 2012: Lebensfäden. Zehn autobiographische Versuche, Heidelberg.

Lebow, Richard Ned 2003: The Tragic Vision of Politics. Ethics, Interests and Orders, Cambridge.

Marlantes, Karl 2010: Matterhorn. A Novel of the Vietnam War, London.

Mead, Walter Russell 2002: Special Providence. American Foreign Policy and How it Changed the World, New York, NY.

Mentzos, Stavros 2002: Der Krieg und seine psychosozialen Funktionen, 2. Aufl., Göttingen.

Morgan, David H. J. 1994: Theater of War. Combat, the Military, and Masculinities, in: Brod, Harry/Kaufman, Michael (Hrsg.): Theorizing Masculinities, Thousand Oaks, CA, 165-182.

Müller, Harald 2008: Wie kann eine neue Weltordnung aussehen? Wege in eine nachhaltige Politik, Frankfurt a. M.

Neusüß, Christel 1989: Die Kopfgeburten der Arbeiterbewegung oder Die Genossin Rosa Luxemburg bringt alles durcheinander, Hamburg.

Russett, Bruce M./Oneal, John R. 2001: Triangulating Peace. Democracy, Interdependence, and International Organizations, New York, NY.

Seifert, Ruth/Eifler, Christine (Hrsg.) 2003: Gender und Militär. Internationale Erfahrungen mit Frauen und Männern in Streitkräften, Königstein.

Senghaas, Dieter 2004: Zum irdischen Frieden. Erkenntnisse und Vermutungen, Frankfurt a. M.

Senghaas, Dieter 2010: Rettung durch den Kleinstaat? Überlegungen zum »AntiLeviathan «-Leitmotiv im Werk von Leopold Kohr, in: Leviathan 38: 2, 251-267. Senghaas, Dieter 2012: Weltordnung in einer zerklüfteten Welt, Frankfurt a. M. Vaget, Hans R. (Hrsg.) 2010: Im Schatten Wagners. Thomas Mann über Richard Wagner - Texte und Zeugnisse, München.

Waldeck, Ruth 1989: Jugend im Nationalsozialismus - ein Erbe unserer Mütter, in: Busch, Hans-Joachim/Krovoza, Alfred (Hrsg.): Subjektivität und Geschichte. Perspektiven politischer Psychologie, Frankfurt a. M., 59-79.

Waldeck, Ruth 1991: »Kassandra« - oder die Lust der Frauen am Krieg der Männer, in: Ethnopsychoanalyse, Bd. 2: Herrschaft, Anpassung, Widerstand, Frankfurt a. M., 16-35.

Wasmuht, Ulrike 1998: »Frieden schaffen, mit Waffen?« - Zur Uneindeutigkeit der

Bellizismus-, Belli-Pazifismus- und Pazifismusdabette, in: Grundmann/Hummel 1998, 19-40.

Welch, David A. 2003: Why International Relations Theorists Should Stop Reading Thucydides, in: Review of International Studies 29: 3, 301-319. 
Weller, Christoph/Zürn, Michael 1991: Das Ende des Militärs? Eine Auseinandersetzung mit »Staat und Krieg«, in: Karl, Wilfried/Nielebock, Thomas (Hrsg.): Die Zukunft des Militärs in Industriegesellschaften (Jahrbuch für Friedens- und Konfliktforschung, Band XVIII), Baden-Baden, 93-107.

\section{Der Autor}

Dr. Gert Krell, em. Professor für Internationale Beziehungen am Fachbereich Gesellschaftswissenschaften der Johann Wolfgang Goethe-Universität in Frankfurt am Main.

E-Mail: gertkrell@arcor.de

Internet-Auftritt: www.gert-krell.de 